\title{
A low-cost, MR-compatible olfactometer
}

\author{
STEVEN B. LOWEN and SCOTT E. LUKAS \\ McLean Hospital and Harvard Medical School, Belmont, Massachusetts
}

\begin{abstract}
We present a design for an olfactometer, suitable for fMRI experiments, that can be constructed at extremely low cost. The olfactometer presents odors directly to the nose via a nasal cannula at unobtrusively low flow velocities, with no large assemblies required on or near the subject's face. The olfactometer can be controlled manually, or by computer via a serial interface. A validation study verified that the olfactometer reliably presents odors to test subjects. Errors and response latency times decreased with increased flow rate in an orderly manner, as expected.
\end{abstract}

Interest in the sense of smell is ancient. The subject appears in some of the first written documents (Pichersky, 2004). Scientific studies of olfaction began in the 19th century (van Harreveld, 2003); the Dutch physiologist Hendrik Zwaardemaker (1857-1930) designed and built one of the first practical olfactometers late in that century. Since then, a plethora of odor delivery devices have appeared.

The reasons for studying odors stem from the understanding that the olfactory system is well developed in a number of animals. Although it is less developed in humans, the fact that the third cranial (olfactory) nerve has a direct connection to the limbic region of the central nervous system suggests that some aspects of detecting odors do play a significant role in certain forms of therapy (Lis-Balchin, 1997), cosmetics (Berliner, Jennings-White, \& Lavker, 1991), and cue-induced craving (Schneider et al., 2001).

Odor delivery devices span a range of applications, both for human subjects and animal preparations, and vary in sophistication and complexity. Hornung and Mozell (1977) described a system that delivered radioactive odorant to a frog preparation in a pulsatile fashion, essentially an artificial sniff. Special precautions were in place to ensure that the radioactive odors did not escape. Another system presented odors in a wide range of precise dilution values for the quantification of olfactory response (Vigouroux, Viret, \& Duchamp, 1988). For precisely metered absolute amounts of odorant, rather than concentrations of odorant in the air flow, Palmer, Stough, and Patterson (1999) described a nebulizer system. Pearce (1997) described an odor delivery system designed to test an artificial olfactory device or "electronic nose." For presentation directly to hen epithelial tissue, another odor delivery system had very rapid rise and fall times, as well as precisely adjustable humidity (McKeegan, Demmers, Wathes, Jones, \& Gentle, 2002). Other olfactometer designs tested the alerting response to odors during sleep

Development of this device was supported by NIDA Grants DA016612 (to S.B.L.) and DA03994 and DA00343 (to S.E.L.). Correspondence relating to this article may be sent to S. B. Lowen, 115 Mill St., Belmont, MA 02478 (e-mail: lowen@mclean.harvard.edu).
(Badia, Wesensten, Lammers, Culpepper, \& Harsh, 1990) or the ability of subjects to identify a target odor with up to 15 other odors presented simultaneously (Jinks \& Laing, 1999). Vigouroux, Bertrand, Farget, Plailly, and Royet (2005) presented a method for manually delivering odors synchronized to a subject's inhalation in an MR environment. Finally, Lorig, Elmes, Zald, and Pardo (1999) described a sophisticated MR-compatible olfactometer that delivers precise amounts of odorant.

Although a number of sophisticated olfactometer designs exist, these devices are quite expensive to construct, with total bills for materials amounting to several thousand dollars. Furthermore, many of the designs, as presented, do not include sufficient detail for researchers to actually construct them. We designed a low-cost olfactometer with many useful features, and here present instructions sufficient to enable relative novices to build and use it.

This article includes a discussion of the design issues of such a device, a detailed description of its operation and construction, the types of odorants used, and a description of the limitations of the system. Also, the design and results of a validation study with a sample of healthy volunteers are presented. Finally, a complete list of parts, sources, and references is provided.

\section{DESIGN}

Our design goals for the olfactometer were as follows: (1) subject safety; (2) simple, low-cost construction and use; (3) magnetic resonance imaging (MRI) compatibility; (4) reliability; (5) odor switching without other concomitant stimuli, such as airflow rate changes; (6) lack of cross-contamination between odorant streams; (7) constant odor delivery rates within and among subjects; (8) easily changeable odorants; and (9) expandability to more odorants.

To satisfy these criteria, we made the following design choices. First, a number of components of the olfactometer, including the pump and especially the solenoid valves, are magnetic, and thus must be located far from the MR scanner. Second, we chose to deliver odors through a nasal cannula, rather than a face mask or sniffing port. This pro- 
vides added flexibility and simplicity, since space in MR scanners is at a premium. Furthermore, nasal cannulas are inexpensive and can be discarded, unlike many masks or sniffing ports, which must be cleaned thoroughly between subjects. Finally, masks can increase feelings of claustrophobia, a frequent problem in MR experiments. However, using a cannula precludes the use of high flow rates, which become unpleasant; subjects reported that rates up to about $0.5 \mathrm{l} / \mathrm{min}$ are unobtrusive. Third, we decided to use only one path from the main apparatus to the subject, to reduce complexity and cost. The use of a single flow regulator and meter ensures that the flow rate remains constant for all odorants, thus eliminating this potential confounding cue when switching among odors. A picture of the finished olfactometer appears in Figure 1.

The combination of low flow rate and a long, single path for the odors requires the use of narrow tubing. Largediameter tubing would constitute a significant volume, and at our relatively modest flow rate the odors would take prohibitively long to reach the subject. Instead, we used tubing with a small, 1/16-in. diameter. With $50 \mathrm{ft}$ of this tubing, at a flow rate of $0.3 \mathrm{l} / \mathrm{min}$, the calculated odorant delay is $6 \mathrm{sec}$ from the onset of solenoid action to delivery of the odor to the subject.

To minimize cost, we designed our olfactometer around liquid-based odorants. However, since nearly any odorant can be dissolved in a liquid, we lose little flexibility by this choice. Furthermore, some odorants, such as beer, prove quite difficult to dispense in other forms. Finally, some odorant preparations, such as small plastic cubes impregnated with peppermint oil (Badia et al., 1990) can be placed in the apparatus and function much like liquidbased odorants. In all cases, the relatively low flow rate helps ensure that the air exiting the odorant bottles carries a high and relatively constant concentration of odorant.

Another trade-off exists between number of potential odorants and cost. We chose to use three odorant bottles.
This setup requires two solenoid valves and provides somewhat more flexibility than the minimum of two bottles, for odorant and placebo (water). We used a multiport connector with six ports, though, to facilitate the addition of more odorants. With only three bottles, we closed the remaining two ports with short metal rods (see Figure 1).

\section{OPERATION}

A schematic diagram of the olfactometer appears in Figure 2, and Figure 3 presents the associated electrical circuit. An aquarium pump (Tetratec DW96-2) supplies air at sufficient pressure to deliver odorants to subjects; we used flows in the range $0.1-0.5 \mathrm{l} / \mathrm{min}$. At the maximum flow rate used, the olfactometer develops a backpressure of 180 torr; the pump is specified to deliver more than twice this flow at the same pressure. As the air exits the pump, it travels through 3/16-in. vinyl tubing until just before the odorant chambers. After the pump, the air stream branches into two paths. An adjustable valve is in each path; one path then exhausts to the room, and the other continues toward the subject. Normally the latter valve remains fully open, and the exhaust valve controls the air flow. This setup minimizes backpressure to the pump, extending its life.

After the valve, the air flows through an activated charcoal filter (Omnifilter R200 or similar), which removes almost any odors which might be in the room air or introduced by the pump. To prevent fine charcoal dust from entering the line, the air is passed through a high-efficiency particle arresting (HEPA) filter assembly (Tiara Medical Systems TBF-200S) immediately after the charcoal filter. We use an inexpensive device designed for continuous positive airway pressure machines. An inexpensive acrylic flow meter with a floating glass ball provides the means to measure the air flow.

The filtered and metered air next passes through a pair of single-pole, double-throw solenoid valves. The second

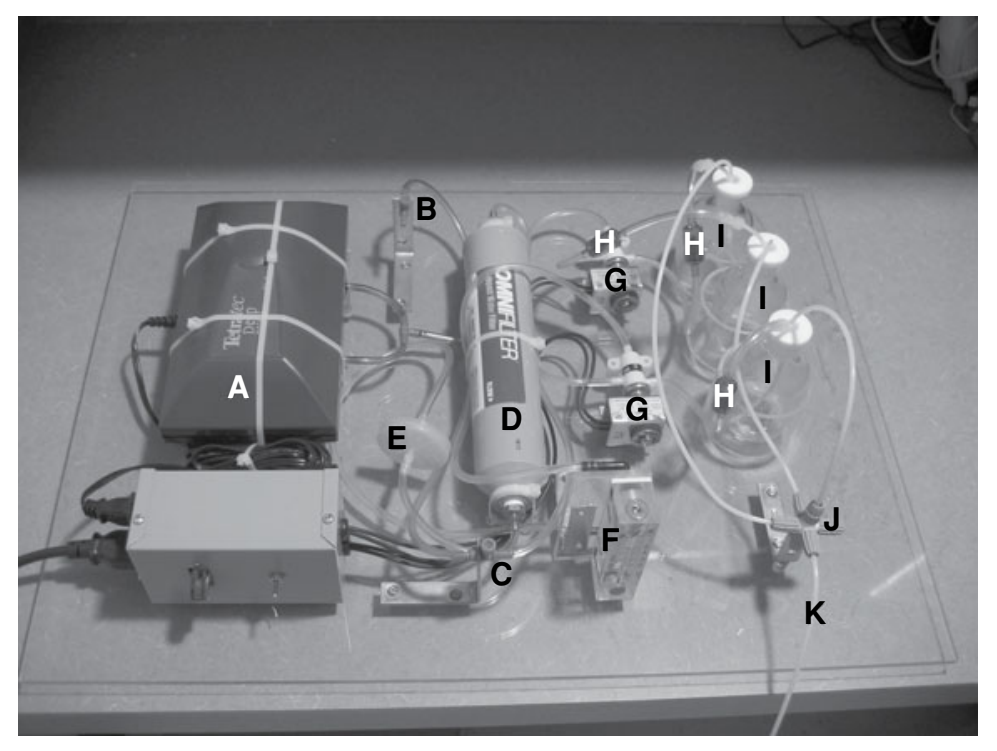

Figure 1.A photograph of the olfactometer. 


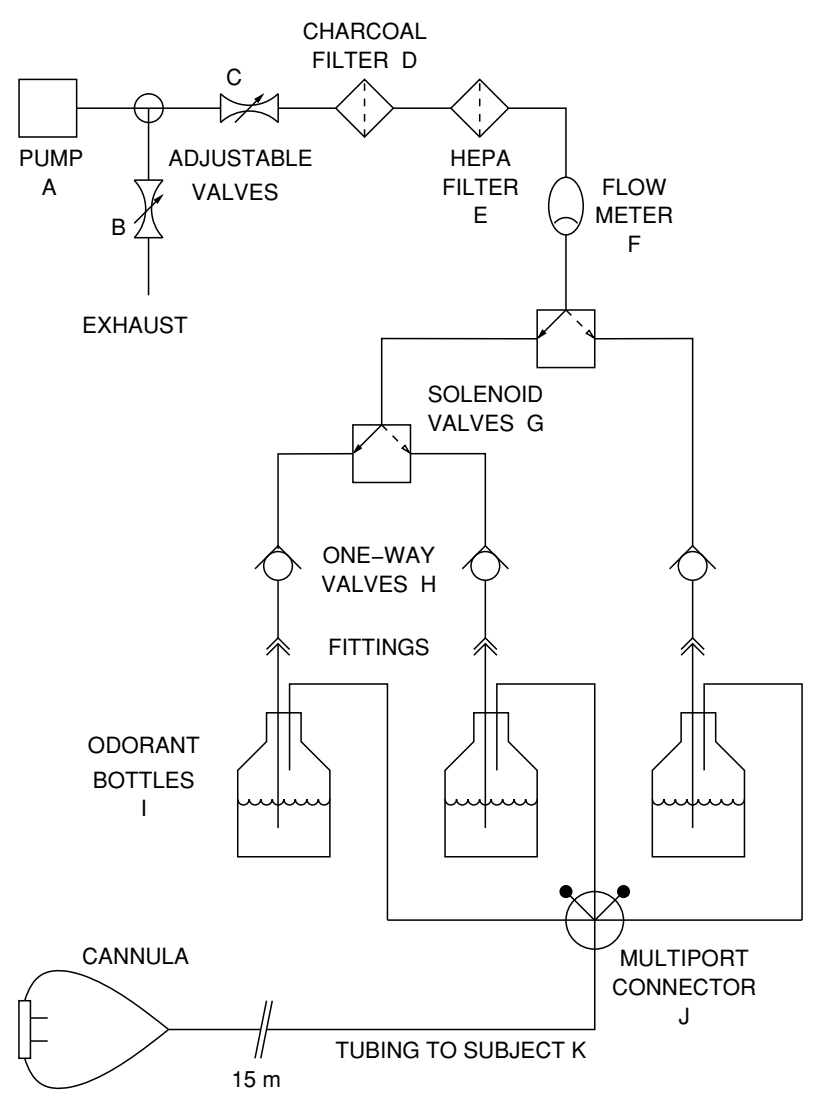

Figure 2. A schematic representation of the olfactometer.

solenoid valve is connected to the normally open output port of the first, so that activating either valve by itself selects a unique output stream. Solenoids were mounted with rubber grommets acting as shock absorbers to reduce the audible clicks generated by these devices during switching. This sound-dampening reduces the auditory cues associated with switching among odors, which is useful for non-MR applications; in an MR environment, such noises would be far below the detectability threshold.
More solenoid valves can easily be added to increase the number of air paths and odorant choices. Each of the three air lines passes through a one-way check valve to block any backflow of odorant into the solenoid valves.

Reducing couplings are used to mate with 1/16-in. inner diameter (ID) polytetrafluoroethylene (PTFE) tubing. With the exception of the short cannula that interfaces with the subject, all remaining parts are either PTFE or glass. This minimizes the chance of adsorption of the odorants onto the olfactometer surfaces and latent release of odors. Each air line ends at the bottom of a glass bottle. The active air stream bubbles through the odorant solution and exits through another air line that begins near the top of the bottle. All three lines converge on a PTFE multiport connector, with a single line emerging, which then travels 10-15 $\mathrm{m}$ into the MRI chamber. The far end of the line then replaces the main feed line of a nasal cannula. With our device, flow measurements at the cannula agree with values displayed on the meter.

\section{Odorants}

Since the olfactometer works by bubbling air at a fixed rate through solutions, odorant concentration as experienced by the subject does not vary in normal use for a given odorant solution. Odor strengths can be decreased by diluting the active ingredient in an odorless solute, such as water for odorants miscible in water, or canola or mineral oil for other odorants. This changes the vapor equilibrium pressure in the odorant bottle. We used vinegar as an odor and found that the standard 5\% strength commercially available was easily detected; diluting this with an equal quantity of water yielded a vinegar odor that was still easily discernible. We chose the diluted concentration, since in pilot studies some subjects reported the higher concentration as being mildly unpleasant. Alternatively, odorant concentration can be changed by adjusting the air flow rate.

The bubbling action can also produce foam in the odorant bottles that, if not contained, could lead to contamination of the tubing. Numerous commercial antifoaming agents exist (including dimethyl polysiloxanes and some

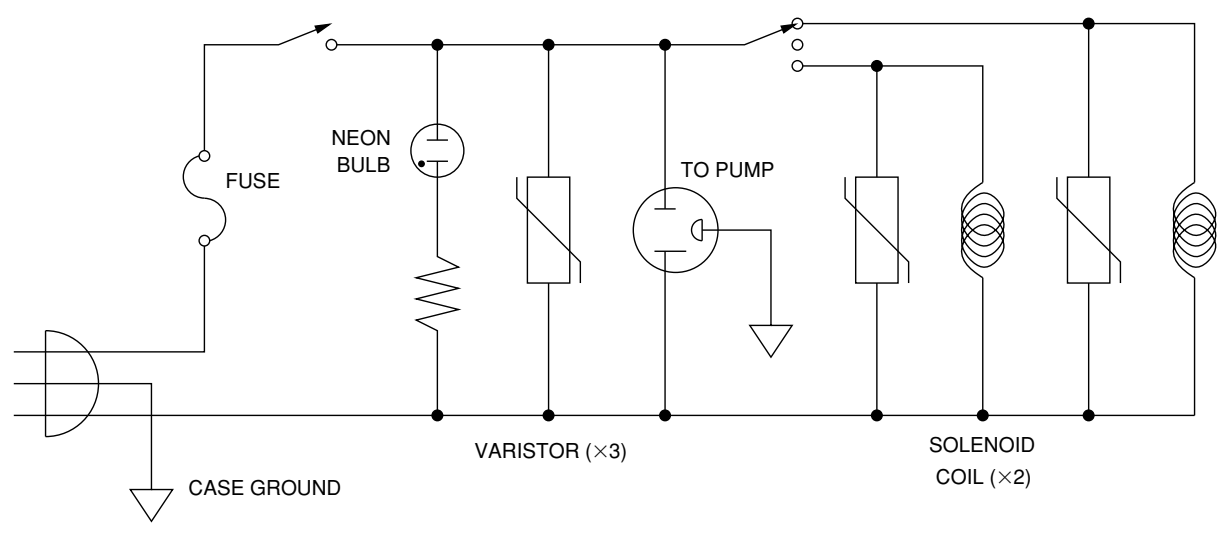

Figure 3. An electrical circuit diagram for the olfactometer. 
stearates), but we found that a thin layer (about $1 \mathrm{ml}$ ) of canola oil reduces the foam. This technique was applied when we tested beer in our olfactometer.

Finally, we included a placebo odorant (water) in the bottle that receives flow when neither solenoid is energized (leftmost bottle in Figure 2). Having water in the default position ensures that active odors will not be presented to subjects when the olfactometer is first turned on.

\section{VALIDATION}

To test the performance of our olfactometer, we conducted the following study.

\section{Method}

Subjects. Ten healthy volunteers, 6 females and 4 males who ranged from 22-55 years old (mean age 33), served as subjects. Ages did not differ significantly between the two sexes (two-tailed nonparametric $p=.59$ ). Another subject (a 33-year-old male) was dropped because of technical problems during the odor presentation. The McLean Hospital Institutional Board reviewed and approved the protocol and informed consent used in the study; all subjects read and signed the informed consent form.

Experimental design. The subjects rested comfortably in a soundproofed test chamber during the study, with a noise generator operating in the room. A disposable nasal cannula was placed in each subject's nostrils, with the other end connected to the PTFE tube emanating from the olfactometer. The cannulas are hospitalapproved for delivering oxygen. The olfactometer and most of the tubing resided outside the chamber. The subjects were continuously monitored by video and audio during the experiments. Instructions were presented to the subjects with a computer monitor, with responses conveyed using a computer mouse. The subjects were alerted via intercom just before the beginning of each olfaction event. All subjects were informed of the experimental protocol, including the nature of the odors and the varying flow rates used. Only the order of odor presentation remained blind to the subjects.

The experiment comprised two protocols, administered in the order listed below, one immediately following the other.

In the first protocol, the subjects were exposed to three odors: beer (Newcastle Brown Ale, with a small amount of canola oil to prevent foaming), limonene (a component of natural lemon and orange scent), and acetic acid (white vinegar mixed with an equal quantity of water, yielding $2.5 \%$ acetic acid) delivered at three flow rates $(0.1,0.3$, and $0.51 / \mathrm{min}) .{ }^{1}$ The subjects received all nine combinations of odor and flow rate in a randomized, counterbalanced order. At the beginning of each event (after they were alerted), the subjects were instructed to click a button on the screen when they first detected the odor. Next followed a random wait time from 0 to $59 \mathrm{sec}$ (uniformly distributed), after which the solenoid was activated and odor flowed into the tubing. If subjects indicated an odor, they were asked to guess which odor was presented (beer, limonene, or vinegar), as well as to report their confidence in their choice. This completed the odor event.

In the second protocol, the subjects received the same odorant (acetic acid) at the same flow rate $(0.31 / \mathrm{min})$ but at four different concentrations: $5 \%, 2.5 \%, 1.25 \%$, and $0 \%$ (pure water or placebo). The $0 \%$ concentration odor event was carried out just like the other concentrations. For completeness, a solenoid click was simulated when the odor would have been turned on, although the air flow path was not altered and continued to flow through the bottle containing water. The subjects received these four concentrations in a random, counterbalanced order. At the beginning of each event (after the initial alert), the subjects were instructed to click a button on the screen when they first detected the odor. Next followed a random wait time from 0 to $59 \mathrm{sec}$ (uniformly distributed), after which the solenoid was activated (or simulated). The subjects were told that one of the trials (with $0 \%$ acetic acid) did not involve presentation of an odorant, and they were instructed to refrain from clicking the button for this trial.

For both protocols, the solenoid was turned off at the conclusion of an event, and a time-out of $3 \mathrm{~min}$ followed. The subjects rested or read during this time-out. If the subjects failed to detect the odor within $2 \mathrm{~min}$, the screen was blanked, the solenoid was turned off, and the event was recorded as a miss. The same 3-min time-out followed in this case as well.

Following the odor experiment, the subjects completed a short visual analogue scale questionnaire to assess their comfort during the study.

\section{Results}

All subjects completed the entire experiment, which lasted between 60 and $90 \mathrm{~min}$. No subjects reported undue discomfort due to either the odors or the apparatus. All subjects were instructed to report immediately any clicks or similar noises throughout the experiment. One subject (no. 4) reported hearing the clicks associated with the solenoid action immediately after the third odor event. Further sound-masking measures were immediately taken (eliminating the sounds for this subject), and these measures were retained for all further subjects. The last subject (No. 10) reported the possibility of faint clicks after the experiment, but was not sure. No other subjects reported any solenoid-related sounds. Recall that this experiment took place outside of an MRI environment; in an MRI scanner, these noises would be undetectable even without the masking measures that we employed.

Subjects correctly identified the odors much better than would be expected for random responses. Table 1 shows the odor identification matrix for this study. Combining incorrect identifications with misses, we had 69 correct odor identifications out of 90 trials with three choices; summing the probabilities of obtaining 69 or more correct identifications, given random responses, yields $p \ll$ $10^{-6}$. A similar approach for the individual odors yields $p<2 \times 10^{-6}$ for beer, $p<10^{-6}$ for limonene, and $p<$ $5 \times 10^{-5}$ for acetic acid. As expected, subjects performed better at higher flow rates, as shown in Table 2 .

After combining incorrect identifications with misses, a chi-square test showed that results for the 0.1 and 0.51 / min flow rates differed significantly (two-tailed $p<$ .002 ). However, comparisons between the $0.3 \mathrm{l} / \mathrm{min}$ flow rate and the other two were not statistically significant. Accuracy did not depend on trial number (first, middle, or last third of Protocol 1) nor on odor history (previous odor identical or not).

Table 1

Odor Identification Versus Presented Odor

\begin{tabular}{lcccc}
\hline & \multicolumn{4}{c}{ Detected } \\
\cline { 2 - 5 } Presented & Beer & Limonene & Acetic Acid & Miss \\
\hline Beer & 23 & 5 & 2 & 0 \\
Limonene & 2 & 25 & 2 & 1 \\
Acetic Acid & 2 & 5 & 21 & 2 \\
\hline
\end{tabular}


Table 2

Odor Identification and Detection Versus Flow Rate

\begin{tabular}{cccccc}
\hline $\begin{array}{c}\text { Flow Rate } \\
(1 / \mathrm{min})\end{array}$ & \multicolumn{3}{c}{ Response Type } & $\begin{array}{c}\text { Latency to Detection } \\
(\mathrm{sec} ; M \pm S E M)\end{array}$ & $\begin{array}{c}\text { Air Travel Time } \\
(\mathrm{sec})\end{array}$ \\
\cline { 2 - 4 } & Correct & Incorrect & Miss & & $\begin{array}{c}\text { and } \\
0.1\end{array}$ \\
17 & 10 & 3 & $41.7 \pm 5.5$ & 18.1 \\
0.3 & 24 & 6 & 0 & $19.7 \pm 3.5$ & 6.0 \\
0.5 & 28 & 2 & 0 & $11.5 \pm 1.6$ & 3.6 \\
\hline
\end{tabular}

As expected, the latency to detection times varied strongly with flow rate. Table 2 presents these results as means \pm standard errors of the mean. Also presented, in the rightmost column, is the expected mechanical delay for odors to propagate through approximately $50 \mathrm{ft}$ of $1 / 16$-in. tubing at the specified flow rates. Since the measured response times greatly exceeded the expected time for odor propagation, most of the delay must be physiological. Lower flow rates correspond to weaker perception of odors, and therefore significantly delayed detection. The delay times have a significantly skewed distribution, so that a nonparametric test of differences among these times is warranted. We employed the two-tailed MannWhitney $U$ test, which yields $p<.008$ when the results for 0.3 and $0.5 \mathrm{l} / \mathrm{min}$ are compared, $p<2 \times 10^{-5}$ for 0.3 and $0.1 \mathrm{l} / \mathrm{min}$, and $p<10^{-6}$ for 0.5 and $0.11 / \mathrm{min}$. We used this test for evaluating significance among all classes of continuous-valued variables, since it represents only a small loss of power over parametric types of analysis. Detection times did not vary significantly with odor type, likely because the flow rate effect dominated odor effects. (Even correcting for the mean detection time by flow rate did not yield a significant odor effect.) Detection times did vary significantly with history, though. Subjects showed a trend toward responding more slowly for repeated odors: $32.8 \pm 6.4$ versus $19.8 \pm 2.7$ (sec; $M \pm S E M)$, respectively, for repeated versus nonrepeated odors, with a twotailed nonparametric significance of $p<.006$.

Subjects reported more confidence in odor identifications that were indeed correct $(75.0 \pm 2.9)$ than in those that were incorrect $(65.4 \pm 5.2)$, but this trend failed to achieve significance.

In Protocol 2, the flow rate and odorant type remained constant $(0.3 \mathrm{l} / \mathrm{min}$ of acetic acid solution) while the concentration of odorant varied. Subjects exhibited excellent accuracy in discriminating between true odorant and placebo, as shown in Table 3, which presents detection accuracies as well as mean latencies to detection times plus or minus standard error. For evaluating significance, a subject performing at chance would randomly choose detection for 30 of the trials, and no detection for the other 10 . The probability of getting two or fewer errors in this case is $p<10^{-6}$. The detection times of our subjects do not show any significant variation with concentration. Taken together, these results suggest that all positive concentrations of acetic acid used were more than sufficient to elicit a response, with no appreciable differences among them. Lower concentrations of acetic acid would presumably yield a more graded response.
Finally, age- and sex-related differences in accuracy, detection time (for correct trials and for all trials), and confidence were evaluated. Spearman's rank order correlation coefficient was used to determine significance for age effects, and the Mann-Whitney $U$ test was used to evaluate differences between the two sexes. No agerelated effects were significant, and only two sex-related measures showed a trend toward significance: Females had faster detection times over all trials $(p<.04)$, and especially with correct trials $(p<.003)$.

\section{DISCUSSION}

As evidenced by the subject responses presented above, the olfactometer reliably presented the specified odors at the appropriate times. Subjects accurately identified the odors within a time frame that was consistent with the rate of delivery. Furthermore, increasing the flow rate resulted in faster and more accurate subject responses. These results were statistically significant to a very high degree. As this was an initial feasibility and validation study, we did not have sufficient power to detect sex- or age-related differences in our data. In keeping with the exploratory nature of the study and our goal of validating the functioning of the olfactometer, many independent statistical tests (23) were conducted, which changed the significance limit from $<.05$ to $<.0023$ because of the multiplecomparison (Bonferroni) correction.

\section{Limitations}

Perhaps the most significant limitation is the relatively long delay between solenoid activation and odorant presentation. We calculated this to be $6 \mathrm{sec}$ at a typical flow rate of $0.31 / \mathrm{min}$ (see Table 2 ). Thus, the present design would not permit the study of odor-event-related potentials. This delay can be reduced but not eliminated (see the Enhancements section below). Shortening the tubing between the main apparatus and the subject would reduce the delay proportionally without increasing the flow rate

Table 3 Identification Versus Concentration

\begin{tabular}{ccc}
\hline $\begin{array}{c}\text { Acetic Acid } \\
\text { Concentration }\end{array}$ & $\begin{array}{c}\text { Number of } \\
\text { Detections }\end{array}$ & $\begin{array}{c}\text { Latency to Detection } \\
\text { (sec; } M \pm S E M \text { ) }\end{array}$ \\
\hline $0.00 \%$ & $2 / 10$ & $45.0 \pm 20.2$ \\
$1.25 \%$ & $10 / 10$ & $25.5 \pm 8.7$ \\
$2.50 \%$ & $10 / 10$ & $28.5 \pm 10.7$ \\
$5.00 \%$ & $10 / 10$ & $19.6 \pm 6.1$ \\
\hline
\end{tabular}


and thereby causing subject discomfort. This would be possible for MR scanners with actively shielded magnets and proportionally smaller fringe fields. Reducing tube diameter would also decrease the delay, at the cost of increased pressure. PTFE tubing is also available in a 1/32-in. ID size, which at half the diameter would quarter the transit time, to about $1.5 \mathrm{sec}$. The backpressure would quadruple, however, necessitating a different pump, and likely new tubing and connectors.

Another disadvantage of the configuration is the possibility of odors adsorbing to the interior surfaces of the multiport connector or the long output tubing during an odor presentation, and then reemerging during presentation of other odors or a placebo. Although this is theoretically possible, all such surfaces are pure PTFE, making this a minor effect.

Our olfactometer is based on using liquids, which has implications for performance. The heights of the water columns in the different odorant bottles represent a varying backpressure against which the pump must push the air, with concomitant variations in flow rate. However, we measured pressure of 77 torr at $0.3 \mathrm{l} / \mathrm{min}$. Even an inch of height difference among the odorant containers would cause a difference of only 1.87 torr. Assuming a roughly linear pressure/flow relationship, that would translate to a flow difference of less than $2.5 \%$, or an absolute flow rate of $0.3 \pm 0.0073 \mathrm{l} / \mathrm{min}$. Such a difference is unlikely to be perceptible.

Another flow-related issue lies in the saturability of the odorants. Consider an odorant that requires a long time to reach equilibrium between its vapor and liquid phases in the odorant bottle. Suppose that this odorant is used only intermittently, with long times between activations in comparison with its equilibrium time. When the corresponding air path is first switched on, the initial air leaving the odorant bottle will be nearly saturated with odorant. Soon after, however, the air passing through the bottle will not have time to achieve equilibrium, and will therefore have a significantly lower concentration of odorant. The low flow rate that we use helps reduce this effect, since it leaves a longer effective time for the vapor to reach equilibrium. The issue could be eliminated entirely by maintaining a constant flow through all odorant bottles and switching the resulting output flows, but switching odors directly would introduce a set of new engineering challenges and design complexities, including disposing of the unused odorant in such a manner that it did not come in contact with the subject's nose. Furthermore, many olfactometers employ variable odorant flow (see, e.g., Lorig et al., 1999).

\section{Enhancements}

The design described above works well for our purposes and fulfills the specified goals; however, a number of modifications can enhance performance, albeit by increasing system cost and complexity. Certainly, the number of odorants can be increased. For more than four odorants, double-pole double-throw solenoid valves can replace some pairs of single-pole double-throw valves.
Multiport connectors exist with at least eight ports, and these could be ganged together if necessary.

To minimize switching times, a separate line could carry odorized air from each odorant bottle toward the subject, with the multiport connector joining the separate air streams close to the subject. After priming each path, this topology would greatly reduce delays with only slightly increased bulkiness and cost.

Although our application does not require activation by computer, this control method can be easily achieved by using software-controlled relay drivers. For future work, we have implemented this technique using Presentation software (www.neuro-bs.com).

\section{REFERENCES}

Badia, P., Wesensten, N., Lammers, W., Culpepper, J., \& Harsh, J. (1990). Responsiveness to olfactory stimuli presented in sleep. Physiology \& Behavior, 48, 87-90.

Berliner, D. L., Jennings-White, C., \& Lavker, R. M. (1991). The human skin: Fragrances and pheromones. Journal of Steroid Biochemistry \& Molecular Biology, 39, 671-679.

Hornung, D. E., \& Mozell, M. M. (1977). Factors influencing the differential sorption of odorant molecules across the olfactory mucosa. Journal of General Physiology, 69, 343-361.

JinKs, A., \& LAING, D. G. (1999). A limit in the processing of components in odour mixtures. Perception, 28, 395-404.

Lis-BALCHIN, M. (1997). Essential oils and "aromatherapy": Their modern role in healing. Journal of the Royal Society of Health, 117, 324-329.

Lorig, T. S., Elmes, D. G., Zald, D. H., \& Pardo, J. V. (1999). A computer-controlled olfactometer for fMRI and electrophysiological studies of olfaction. Behavior Research Methods, Instruments, \& Computers, 31, 370-375. (See also an update at psych.wlu.edu/cnl/ olfactometer_construction.htm)

McKeegan, D. E., Demmers, T. G., Wathes, C. M., Jones, R. B., \& Gentle, M. J. (2002). Stimulus-response functions of single avian olfactory bulb neurones. Brain Research, 953, 101-111.

Palmer, B. R., Stough, C., \& Patterson, J. (1999). A delivery system for olfactory stimuli. Behavior Research Methods, Instruments, \& Computers, 31, 674-679.

PeArce, T. C. (1997). Computational parallels between the biological olfactory pathway and its analogue "the electronic nose": Part II. Sensor-based machine olfaction. Bio Systems, 41, 69-90.

Pichersky, E. (2004). Plant scents. American Scientist, 92, 514-521.

Schneider, F., Habel, U., Wagner, M., Franke, P., Salloum, J. B., Shah, N. J., ET AL. (2001). Subcortical correlates of craving in recently abstinent alcoholic patients. American Journal of Psychiatry, 158, 1075-1083.

VAN HARREVEld, A. P. (2003, October). Odor regulation and the history of odor measurement in Europe. Paper presented at the International Symposium on Odor Measurement, Tokyo.

Vigouroux, M., Bertrand, B., Farget, V., Plailly, J., \& Royet, J. P. (2005). A stimulation method using odors suitable for PET and fMRI studies with recording of physiological and behavioral signals. Journal of Neuroscience Methods, 142, 35-44.

Vigouroux, M., Viret, P., \& Duchamp, A. (1988). A wide concentration range olfactometer for delivery of short reproducible odor pulses. Journal of Neuroscience Methods, 24, 57-63.

\section{NOTE}

1. Both protocols included three odorants and a placebo. We achieved this by switching odorant bottles near the beginning of the breaks between events. In Protocol 1, the placebo was used to separate the active presentations and was not itself presented as a trial. Air lines to the bottles were wiped carefully when the bottles were switched, in order to prevent contamination. 
APPENDIX

Parts List A complete listing of parts and sources follows. We purchased some of the less critical parts at local hardware
stores rather than at the sources listed below, but either should suffice. Many parts can be substituted. Our total
bill for materials was just over US $\$ 400$.

Air Path

A Pump: Tetratec DW96-2 aquarium air pump $\$ 59.99$ at www.petsmart.com

Vinyl tubing

$\$ 4.49$ for $25^{\prime}$ at www.petco.com

B \& C Connector/valve kit \$2.69 at www.petco.com

D Charcoal filter: Omnifilter R200 water filter $\$ 8.99$ at Lowe's Home Improvement Warehouse Associated fitting: Cole-Parmer EW-06450-20

$\$ 3.50$ for a package of 10 at www.coleparmer.com

E High-efficiency particle arresting (HEPA) filter: Tiara Medical Systems TBF-200S

$\$ 16.75$ for a package of 5 at www.tiaramed.com

F Flow meter: Cole-Parmer EW-32460-40

$\$ 41.00$ at www.coleparmer.com

Associated fitting: Cole-Parmer EW-06451-10

$\$ 5.50$ for a package of 10 at www.coleparmer.com

G Solenoid valve: Powers electric/pneumatic air valve 265-1008

$\$ 70.00$ for 2 at Boston Aircontrols, Burlington, MA

H Check valve: Profile check valve

$\$ 5.37$ for 3 at www.petco.com

Reducing connectors: Cole-Parmer EW-30703-46

$\$ 14.75$ for a package of 10 at www.coleparmer.com

Polytetrafluoroethylene (PTFE) tubing: Norton AXH00002 (Fisher Scientific 14-169-15A)

$\$ 37.42$ for a package of 50' at www.fishersci.com

I Odorant bottle: Kimble 15097100 (Fisher Scientific 02-920-1A)

$\$ 17.10$ for a case of 6 at www.fishersci.com

J Multiport connector: Cole-Parmer EW-06473-03 four-way connector

$\$ 66.00$ at www.coleparmer.com

(We actually used the EW-06473-04 six-way connector, which is $\$ 84.00$.)

Main board: Acrylic sheet 24" $\times 24^{\prime \prime}, 1 / 4^{\prime \prime}$ thick, Model 44361

$\$ 16.42$ at www.usplastic.com

Bottle holders: Acrylic tube, 2 1/2" ID, 2 3/4" OD, 6', Model 44041

$\$ 15.36$ at www.usplastic.com

Feet for main board: Clear extruded acrylic rod, 1/2" diameter, 6', Model 44656

$\$ 2.22$ at www.usplastic.com

Supports: $2^{\prime \prime}$ angle brackets

$\$ 2.49$ for a package of 10 at www.homedepot.com

Electrical

Metal enclosure: Newark 91F706/CU-2106-B two-piece painted aluminum minibox

$\$ 6.36$ at www.newark.com

Power cord: Newark 36F1821/17237-B1-8 three-wire power supply cord

$\$ 1.23$ at www.newark.com

Socket: Newark 92N4145/2142 snap-in AC power outlet receptacle

$\$ 1.03$ at www.newark.com

Power switch: Newark 89C8962/PRASA1-16F-BB000 on-off rocker switch

$\$ 0.70$ at www.newark.com

Odorant selector: Newark 61F1245/MTA-106D on-none-on switch

$\$ 3.85$ at www.newark.com

Fuse: Newark 87F1593 GMC 500-mA time-delay fuse

$\$ 0.61$ at www.newark.com

Fuse holder: Newark 91F4760 in-line fuse holder

$\$ 1.25$ at www.newark.com

Varistors: Newark 09F1981 130-VAC metal oxide varistor

$\$ 1.14$ for 3 at www.newark.com

Indicator lamp: Newark 93F3576 neon indicator with built-in resistor, 115 VAC

\$2.98 at www.newark.com

(Manuscript received February 25, 2005;

revision accepted for publication May 13, 2005.) 